\title{
Role of Multi Detector CT in Diagnosis of Small and Large Bowel Obstruction
}

\begin{abstract}
Department of Radiology, Shibin Elkom Teaching Hospital, Menofeya, Egypt. ${ }^{\mathrm{b}}$ Department of Radiology, Benha faculty of medicine, Benha University, Egypt.
\end{abstract}

Hagar A. Abdellatef ${ }^{a}$, Ahmed F. Yousef ${ }^{b}$, Hamada M. Khater ${ }^{\text {b }}$, Adel Abdel Rahman ${ }^{\text {a }}$
Correspondence to: Hagar A. Abdellatef Department of Radiology, Shibin Elkom Teaching Hospital, Menofeya, Egypt.

Email:

hagerbadawy88@gmail.com

Received: 14 September 2019

Accepted: 12 November 2019

\begin{abstract}
Background: A prospective study of 50 patients presented in emergency department with features suggestive of intestinal obstruction which is a common cause for acute abdomen, Aim: To evaluate role of MDCT in diagnosis of bowel obstruction. Methods: 50 patients presented with symptoms and signs suggesting primary diagnosis of intestinal obstruction between July 2017 to June 2019, 24 patients were males and 26 females, with the mean of 46.5 years old. All of them underwent MDCT study on abdomen and pelvis with coronal and sagittal reformatted images to assess presence of bowel obstruction. Results: 40 patients have provisional diagnosis with MDCT as intestinal obstruction, 32 cases were taken for exploratory laparotomy and 8 patients were managed conservatively. $31 \backslash 32$ patients approved to have intestinal obstruction while one case not approved with
\end{abstract} sensitivity and specificity $100 \%$ and $91.4 \%$ respectively. The small bowel is involved in (20\39) $51.28 \%$ of cases, whereas large bowel is involved in (19\39) $48.71 \%$ of cases. In 8 patients, the sign of ischemia seen on MDCT scan and confirmed on exploration, so sensitivity and specificity in diagnosis of bowel ischemia was 100\%. Eight cases diagnosed with MDCT as closed loop, 7/8 approved intra -operatively, only one 6case not approved. so sensitivity and specificity of MDCT in diagnosis of closed loop was (100\% and 96.1\%) respectively. Conclusion: MDCT is highly sensitive and specific in determining the presence or absence of bowel obstruction.

Key words: Small and large bowel obstruction. Bowel ischemia. Closed loop obstruction.

Abbreviation: MDCT= Multiple Detector Computed Tomography 


\section{Introduction:}

Intestinal obstruction is one of the most common emergencies in general surgery and is a major cause of morbidity and financial expenditure worldwide. [1] Different mechanisms and causes of small and large bowel obstruction are noted. These include extrinsic causes (adhesions, closed loop, strangulation, hernia, extrinsic masses [carcinoid tumors, lymphoma, peritoneal carcinomatosis, appendicitis, diverticulitis]), intrinsic causes (adenocarcinoma,

Crohn's disease, tuberculosis, radiation enteropathy, intramural hemorrhage, intussusception), intraluminal causes (e.g., bezoars), and intestinal mal rotation. Large bowel causes include carcinoma, fecal impaction as well as cecal and sigmoid volvulus. [2] Early diagnosis of intestinal obstruction is comparatively difficult.[1] Radiologists have a crucial role in clinical decision making in cases of bowel obstruction by providing answers to specific questions that significantly affect management. [2] The first imaging procedure used in patients with bowel obstruction is conventional radiography with 46-80\% accuracy in determining the presence of obstruction. [3] On ultrasonography, bowel obstruction is considered to be present when dilated loop measures $>2.5 \mathrm{~cm}$ and length of segment is $>10 \mathrm{~cm}$. The etiology can sometimes be determined, but ultrasonography is less accurate than computed tomography (CT), When dilute barium is used for the luminal contrast, the obstructing segment can usually be localized and characterized as complete or incomplete [4]

Unlike oral contrast radiography, which provides imaging of only the luminal surface, CT allows imaging of the abdominal contents outside the lumen. Because of this advantage, the nature of the obstruction, especially when secondary to an extra luminal or intramural malignant process, can be established. Additional abdominal pathology, such as the presence of nodal or liver metastases, ascites, and solid organ parenchymal abnormalities, can often be identified, thereby helping to define the cause of the obstruction [4]. Intravenous contrast helps in diagnosing strangulation, identifying the specific cause of small bowel obstruction and characterizing other pathology such as superior mesenteric artery occlusion or superior mesenteric vein thrombosis, which can produce ileus that mimics mechanical obstruction. [5] 


\section{Patient and methods}

A prospective study of 50 patients presented in presented in emergency department with features suggestive of intestinal obstruction, They were referred to the Radiology Department, Shibin Elkom Teaching Hospital, in the period from July 2017 to June 2019. These patients were referred for radiological assessment complaining from one or more of the following symptoms: vague abdominal pain, absolute constipation, recurrent vomiting and distention. Full clinical assessment including recording of age, sex and presentation were done. Revision of the patient's laboratory investigations including renal function tests (blood urea and serum creatinine), and the radiological \& endoscopic diagnostic investigations previously done for the patients, were reported. Patients were scheduled to undergo MSCT and the results were compared to the operative results when surgery done. The results were also compared to laboratory and histopathology results.

All patients were examined in this study by using a four channel multi-slice CT scanner. (Toshiba machine) with No specific preparation prior to the examinationFourteen patients scanned without contrast,
A large bore (18-gauge) intravenous line was placed in the cubical fossa in 28 patients. IV contrast medium of nonionic contrast (omnipaque) according to the body built ( $1.5 \mathrm{ml} / \mathrm{kg}$ body weight) was given by infusion pump at rate $3 \mathrm{ml} / \mathrm{second}$. Oral contrast medium administration in 12 patients in the form of administration of 50 $\mathrm{ml}$ of omnipaque diluted in 1.5 liter of water, patient was asked to drink diluted contrast over 6 hours prior to examination . Rectal enema was done in 26 patients with diluted omnipaque by insertion of a catheter through rectum. The patient lies supine on the examination table and instructed to follow the breath hold technique. Images were obtained from the diaphragm superiorly down to the symphysis pubis as determined from the scout image. Portal phase images were obtained usually 30 to 40 seconds after initiation of IV contrast. All imaging was performed with slice collimation $2.5 \mathrm{~mm}$, Pitch 1 to 1.5 , matrix $512 \times 512,200$ to 350 milli amper and 120 to140 kilovolt. Total scanning time of 20 to 30 seconds on the 4 channel multi-slice CT scanners, was followed. From this data set, the technologist will generate a set of axial 3 $\mathrm{mm}$ sections and a set of $3 \mathrm{~mm}$ thick coronal, sagittal and oblique multi-planar 
reformatted images at $3 \mathrm{~mm}$ intervals encompassing the entire bowel. All procedures are approved by ethical committee of Faculty of Medicine- Benha University and Shebeen Elkom teaching hospital.

\section{Statistical analysis:}

Data analysis was done using SPSS program version 20. Data presented as number and percentage. Sensitivity, specificity, positive predictive value (PPV), negative predictive value (NPV) and accuracy was calculated.

\section{Results}

This study was carried out on 50 patients The age of patients ranged from 18 to 82 years old with the mean of 46.5 ys old. The study showed prevalence of intestinal obstruction in Males (48\%) in comparison to females $(52 \%)$. All patients have abdominal pain (100\%), Thirty -eight patients presented with recurrent vomiting (76\%), while thirty patients presented with absolute constipation (60\%). By clinical examination $35(70 \%)$ patient have tenderness, while 28 $(56 \%)$ patients have guarding and rigidity.

At current study 40\50 patients were diagnosed with MDCT as intestinal obstruction, 39 of them confirmed to have bowel obstruction, while one case not approved Table 1\&2)

Among 39 cases approved to have bowel obstruction $20(51.28 \%)$ cases were due to small bowel obstruction while $19(48.71 \%)$ cases were due to large bowel obstruction.

Twenty out of thirty nine patients were diagnosed to have SBO causes including adhesions $30 \% \quad(6 / 20)$ incarcerated hernia $25 \%(5 / 20)$ intussusception $15 \%(3 / 20)$, vascular lesions $10 \% \quad(2 / 20)$, one patient was gall stone ileus, one due to ileus, one due to volvulus of small bowel and one case was diagnosed as Crhon's . Large bowel obstruction cases were 19/ 39 (48.7\%), adenocarcinoma was the most common cause of large bowel obstruction with percentage (12/19) $63.15 \%$, while $(5 / 19)$ $26.31 \%$ was due to acute pseudo colonic obstruction. only one case was due to incarcerated para umbilical hernia of colon and another one was due to colico colic intussusception. Among 40 cases diagnosed with MDCT as intestinal obstruction 32 cases managed surgically while 8 cases conservative treatment . 8/32 cases were reported with MDCT to have ischemia of bowel, all of them approved intra-operative (Tables 3\&4) 
Among 32 cases which have provisional diagnosis of bowel obstruction and managed surgically, 8 cases were diagnosed with CT as closed loop obstruction, 6 cases due to incarcerated hernia, one due to adhesion and one due to volvulus of small bowel loop.

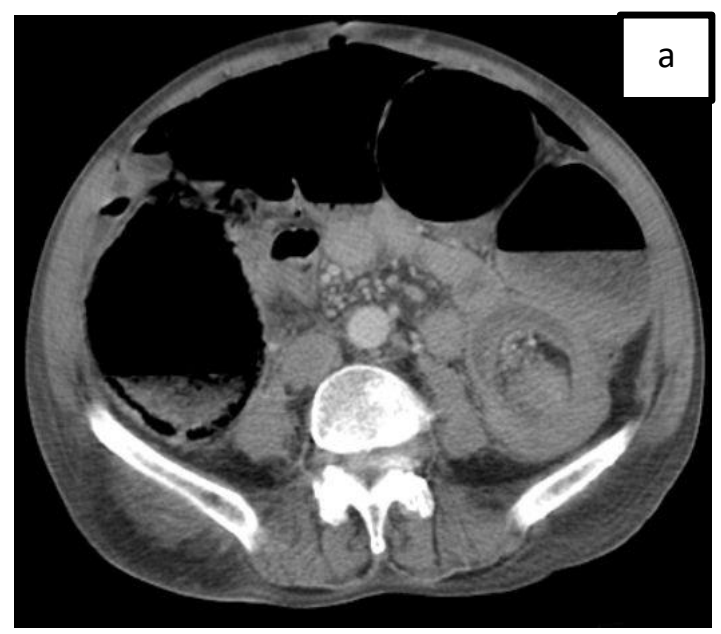

Intra-operative 7 cases of 8 approved to have closed while one case was simple adhesions not closed loop. (Tables 5\&6) .

Fig. 1: (a) colico colic intussusception on amass, (b): coronal reformatted show claw sign
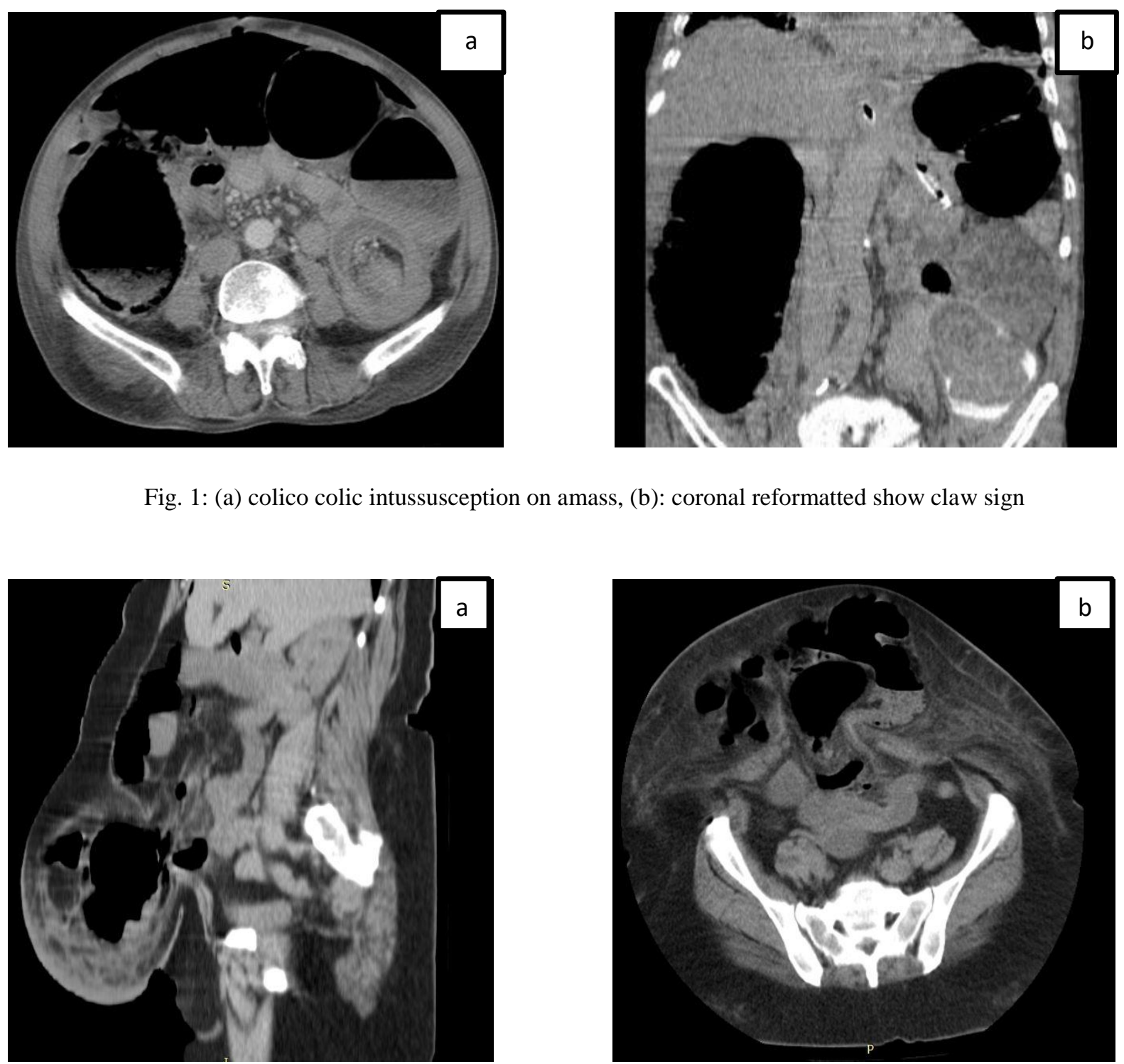

Fig.2: (a) sagittal view show large para umblical incarcerated hernia (b): large hernial defect 

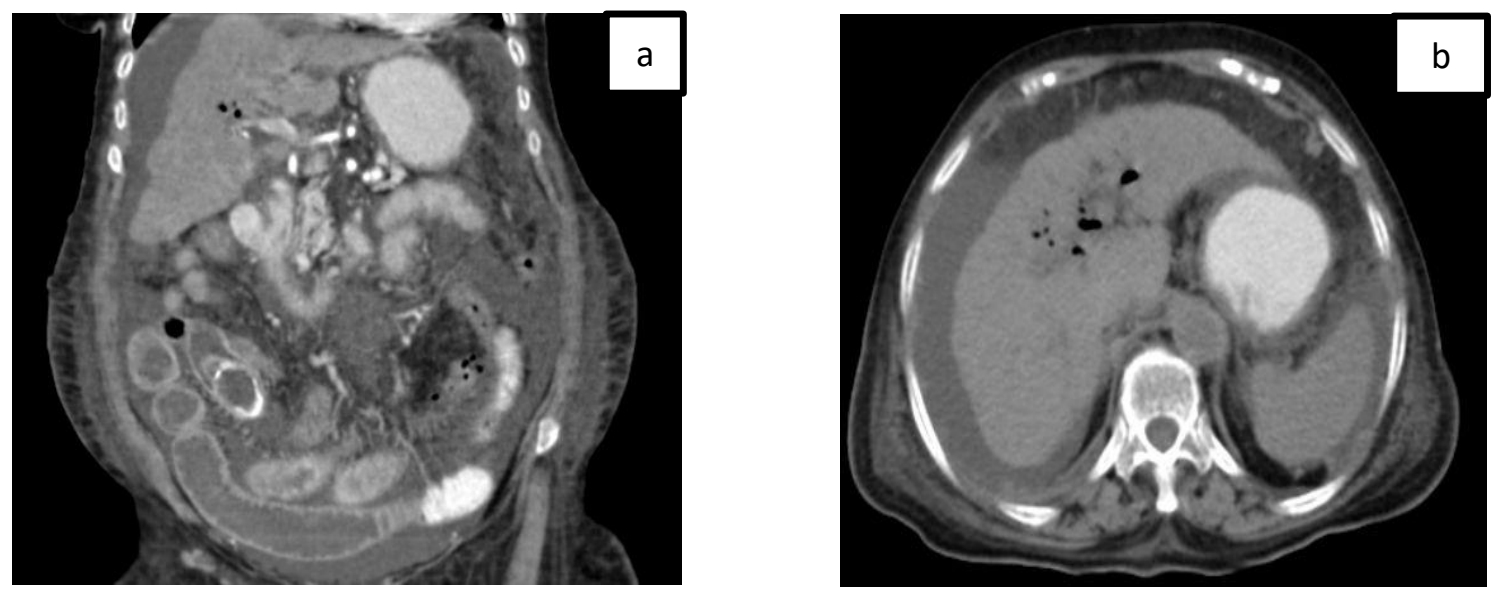

.Fig.3: (a) gall stone illeus (b): axial cut shows airobilia and ascites
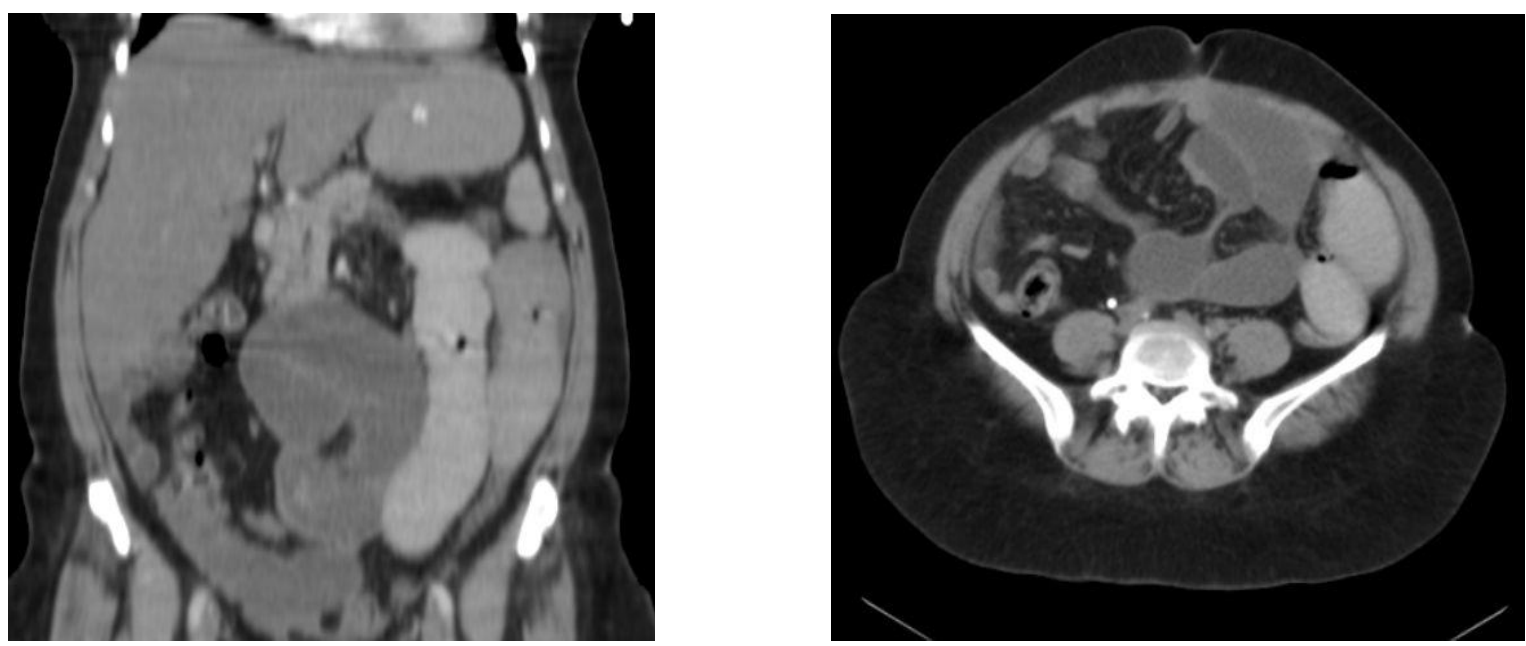

Fig.4: (a) coronal view show closed loop obstruction due to small bowel volvolus (b): note that, two transition zones

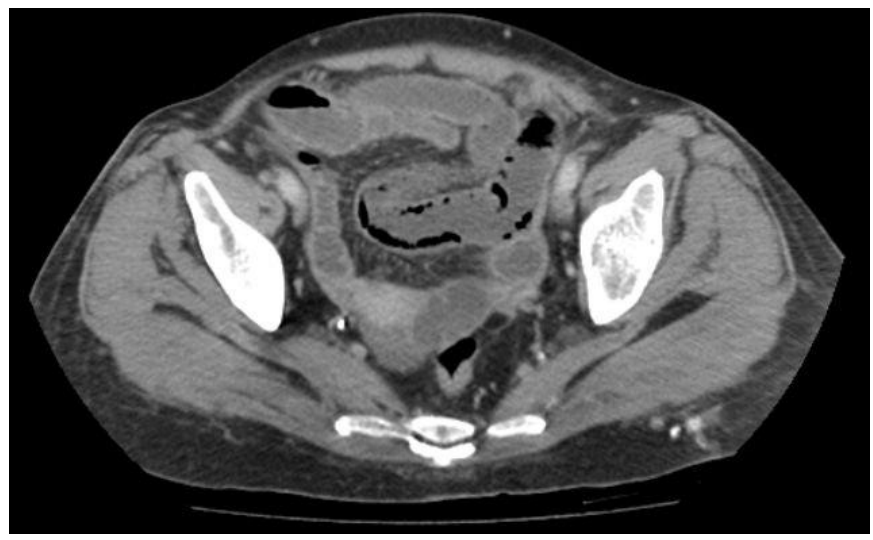

Fig. 5: mesenteric vascular occlusion complicated with pneumatosis intestinalis. 


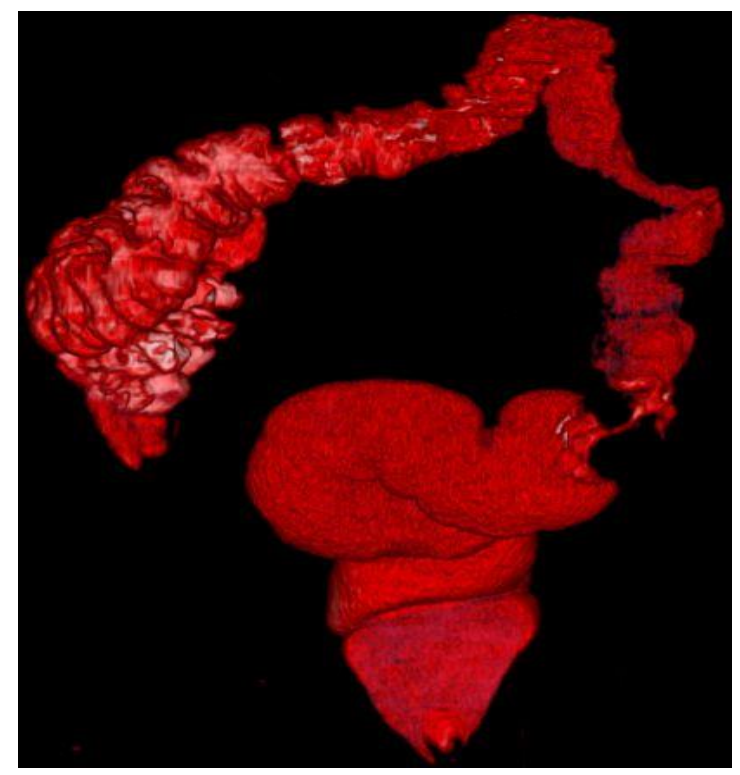

Fig. 6: 3d reconstruction show cancer colon .apple core appearance.

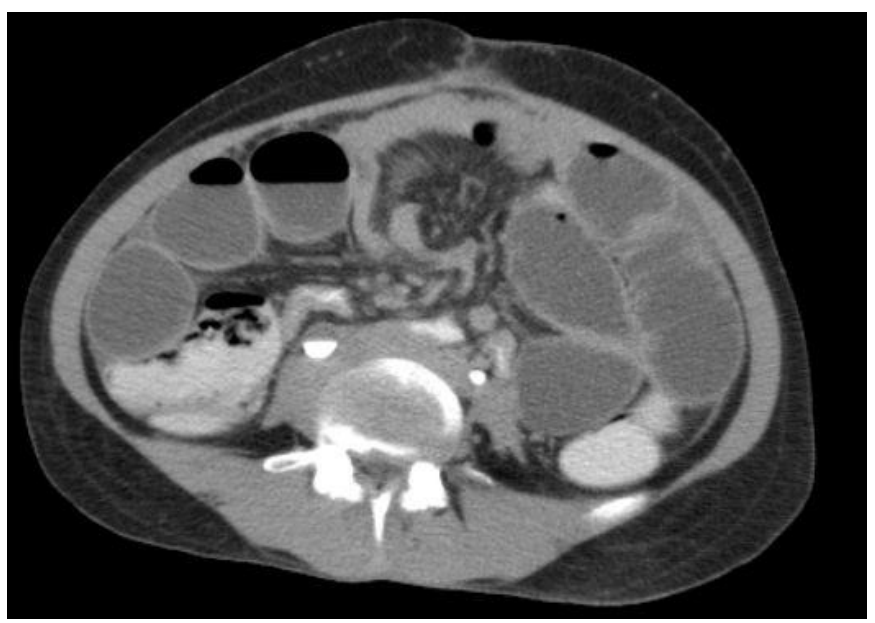

Fig. 7: adhesions of small bowel

Table 1: MDCT results for IO in comparison with approved results.

\begin{tabular}{lll}
\hline & MDCT & Approved results \\
\hline Yes & 40 & 39 \\
No & 10 & 11 \\
\hline
\end{tabular}

Table 2: Validity of MDCT in diagnosis bowel obstruction

\begin{tabular}{lllll}
\hline \multicolumn{4}{l}{ Validity of } & MDCT in diagnosis bowel obstruction \\
\hline Sensitivity & Specificity & PPV & NPV & Accuracy \\
100.0 & 91.6 & 97.5 & 100.0 & 98.00 \\
\hline
\end{tabular}


Table 3: CT results in diagnosis of bowel ischemia VS intra operative results

\begin{tabular}{lcc}
\hline & CT RESULTS & INTRA \\
\hline Yes & 8 & 8 \\
No & 24 & 24 \\
\hline
\end{tabular}

Table 4: Validity of CT in detecting ischemia

\begin{tabular}{lllll}
\hline \multicolumn{1}{l}{ Validity of CT in detecting ischemia } & & \\
\hline Sensitivity & Specificity & PPV & NPV & Accuracy \\
$\mathbf{1 0 0 . 0}$ & 100.0 & 100.0 & 100.0 & 100.0 \\
\hline
\end{tabular}

Table 5: CT results in diagnosis of closed loop obstruction VS intra operative results

\begin{tabular}{lcc}
\hline & CT RESULTS & INTRA \\
\hline Yes & 8 & 7 \\
No & 24 & 25 \\
\hline
\end{tabular}

Table 6: Validity of CT in detecting closed loop

\begin{tabular}{lllll}
\hline \multicolumn{4}{l}{ Validity of CT in detecting closed loop } \\
\hline Sensitivity & Specificity & PPV & NPV & Accuracy \\
$\mathbf{1 0 0 . 0}$ & 96.1 & 87.5 & 100.0 & 96.9 \\
\hline
\end{tabular}

\section{Discussion}

Intestinal obstruction usually occurs acutely and progresses rapidly; therefore, it is critical to devise a treatment plan as early as possible. However, early intestinal obstruction is difficult to diagnose owing to its complexity and variable presentation. [6] Computed tomography plays a crucial role in a correct diagnosis of bowel obstruction (BO). Indeed, computed tomography can confirm a diagnosis of $\mathrm{BO}$, and identify the location and cause of the obstruction. [7] The current study group comprised of 50 patients, who presented to the emergency room (ER) or out-patient department (OPD). All the patients had pain in abdomen while vomiting and constipation were present in $76 \%$ and $60 \%$ patients respectively. On clinical examination tenderness was found in $70 \%$ of patients, guarding/rigidity in $56 \%$ of patients. All patients had their MDCT 
scan. 40 patients diagnosed with MDCT to have bowel obstruction, 39 of them approved either surgically or with follow up while one case not approved surgically, it was passable stricture. Twenty out of thirty nine patients were diagnosed to have SBO causes including adhesions 30\% (6/20), incarcerated hernia $25 \% \quad(5 / 20)$ intussusception, $15 \%$ (3/20), mesenteric vascular occlusion $10 \% \quad(2 / 20)$, one patient was diagnosed as due to gall stone ileus, one due to pancreatitis complicated with multiple liver abscesses and paralytic ileus, one due to volvulus of small bowel and one case diagnosed as Crhon`s. In D.K. Saini et al., 2013 study (24.13\%) patients showed adhesions as cause of obstruction on MDCT. In (10.34\%) patients, hernia was found as a cause of obstruction on CT scan [8]. Miller and colleague 2000 in their study found hernia as a cause of intestinal obstruction in $15 \%$ patients. Due to increasing trend towards early surgical intervention in hernia cases, the incidence of hernia as the cause of obstruction is declining in developed countries.[9] In the current study large bowel obstruction cases were 19/39 (48.7\%), adenocarcinoma was most common cause of large bowel obstruction with percentage (12/19) $63.15 \%$, while (5/19) $26.31 \%$ was due to acute pseudo colonic obstruction. Only one case was due to incarcerated para umbilical hernia of colon and another one was due to colico colic intussusception on a mass. Beattie and co-workers (2007) in their study found that $50 \%(22 / 44)$ of patients had proven mechanical acute LBO, $81 \%$ of whom $(18 / 22)$ had an obstructing carcinoma 2 had a diverticular stricture, 1 had endometriosis and 1 had a sigmoid volvulus.[10]. Another study (11) reported that colorectal cancer was (192/234) cases representing 82.0\%, extracolonic cancer was (11/234), 4.7\%, diverticular disease (4/234) $1.7 \%$, hernia (6/234) $2.5 \%$, and others $8.9 \%$. It was concluded that: LBO is four to five times less common than SBO in the United States. Obstructing colonic malignancy is by far, the most common cause of LBO (>60\%), followed by colonic volvulus and diverticulitis, and less commonly hernias, inflammatory bowel disease, extrinsic compression, and intraluminal contents causing obstruction [11]. In 8 patients, the sign of ischemia seen on MDCT scan and confirmed on exploration. Causes of ischemia in these cases were 6 cases incarcerated hernia complicated with strangulation, presence of hyperdence sign in the wall of entrapped bowel also thickening at bowel wall was diagnostic, 
stranding of fat and minimal free fluid were additional signs. There was one case of superior mesenteric vein thrombus and one case due to mesenteric vascular occlusion with pneumatosis intestinalis from intramural gas produced by bacteria; absence of enhancement with intramural contrast because of vascular hypo perfusion; hazy or streaky mesentery or dirty fat from inflammatory infiltration; and ascitis present. Out of eight cases diagnosed with MDCT as closed loop bowel, six cases were due to incarcerated hernia, one case due to volvulus and one case due to adhesions, 7/8 approved intra-operatively to have closed loop only one case was diagnosed as simple adhesions not closed loop so sensitivity and specificity of MDCT in diagnosis of closed loop bowel obstruction in this study is (100 $\%$ and $96.1 \%$ ) respectively. In a study done on 2004 (12), one hundred fifty patients with CT reports including "small bowel obstruction" were examined to determine the presence of closed loop obstruction (CLSB) and correlate the results with intraoperative findings. Eighty-eight of 150 patients underwent operative intervention for SBO and 24/88 was considered CLSB. With correlation with intraoperative results, sensitivity and specificity for CL-SBO across different readers was $53 \%$ and $83 \%$.
They mention that CT can be helpful in excluding a closed loop component in patients with SBO. As the high negative predictive value (NPV) of $83 \%$ implies that radiologists can exclude closed loop obstruction [12], sensitivity and specificity of MDCT scan for diagnosis of bowel obstruction came out to be $100 \%$ and $91.6 \%$ respectively with accuracy $98 \%$ in all cases ,CT can diagnose presence or absence of mechanical obstruction so the decision of doing urgent surgery or only conservative treatment for patients can be made easy. Liu Jun(2015 A retrospective comparative study was performed to detect the diagnostic value of three imaging modalities for intestinal obstruction, supine and upright (or decubitus) plain abdominal radiography, contrast radiography using Gastrografin, and 64 multi-slice spiral CT (MSCT) (1). A total 142 patients with intestinal obstruction were examined. The diagnostic accuracy of MSCT for detecting small bowel obstruction was $92 \%$ [1]. Erik K. Paulson et al.2015 Another research documented that plain films are less sensitive and less specific method of diagnosing bowel obstruction (13). It was mentioned that: Multi detector CT has been proven to be the single best imaging tool for evaluating patients suspected of having SBO, with sensitivity 
and specificity of $95 \%$; it is also highly accurate in detecting the complications of SBO (13). CT scan should be considered as the primary test for patients with suspected bowel obstruction.[13]. However other authors have reported sensitivity and specificity of MDCT scan in diagnosis of bowel obstruction as high as $94 \%$ and $96 \%$ respectively. This discrepancy could be due to patient selection in these studies favoring patients with higher grades of obstruction. In low-grade obstruction, significance of mild or localized dilatation may be overlooked, adversely affecting the sensitivity and specificity of MDCT scan in diagnosis of bowel obstruction. Attention to this relatively subtle finding may increase the accuracy of MDCT scan in diagnosis of bowel obstruction.

\section{Conclusion:}

MDCT is highly sensitive and specific in determining the presence of bowel obstruction it also demonstrates the site and cause of obstruction, has high sensitivity in bowel ischemia, it plays crucial role in differentiation between mechanical and pseudo bowel obstruction, so it helpful in taking decision at management of cases.

(7)Massimo Galia*,Francesco Agnello ,Ludovico La Grutta,Giuseppe Lo Re, Giuseppe
- MDCT should be recommended for patients with suspected bowel obstruction when clinical and conventional radiographic findings remain indeterminate or strangulation is suspected.

- MDCT has high sensitivity in diagnosing special type of bowel obstruction (Closed loop obstruction) .

\section{References}

(1)Liu Jun1 \& Sun ChangYi1 (2015): diagnostic value of plain and contrast radiography, and multi-slice computed tomography in diagnosing intestinal obstruction in different locations. received: 5 January 2015 /accepted: 6 April 2015 \# association of surgeons of India.

(2) Catarina Silva Ana, Pimenta, Guimarã es Lu1's S (2009). Small bowel obstruction: what to look for.RadioGraphics; 29:423-39.

(3) Herlinger H, Rubesin SE, Morris JB (2000). Small bowel obstruction. In: Gore RM, Levine MS, editors. Textbook of gastrointestinal radiology. 2nd ed. Philadelphia, Pa: Saunders; . p. 815-37.

(4) Durgesh K,Poras CH,Chikkala K,Kiran S (2013) licensee pagepress, italy clinics and practice 2013; 3:e20 doi:10.4081/cp.2013.e20.

(5) Frager D.(2002) Intestinal obstruction role of CT.Gastroenterol Clin North Am 2002;31:777-99.

(6)Antonsen J, Tilma J (2014) Images in clinical medicine. Mechanical small-bowel obstruction. N Engl J Med 28:371-379

Cabibbo,EmanuelelGrassedonio,Bruno Giuseppe Gioia, Gianvincenzo Sparacia ,Antonio Lo 
Benha medical journal vol. 38, special issue (Radiology), 2021

Casto,Roberto Lagalla and Massimo (2015): Midiri:Computed tomography of bowel obstruction: tricks of the trade Expert Rev. Gastroenterol. Hepatol. Early online, 1-11

(8)Durgesh Kumar Saini, Poras Chaudhary,Chikkala Kanak Durga, Kiran Saini (2013) Role of multislice computed tomography in evaluation and management of intestinal obstruction .licensee page press, italy clinics and practice 2013; 3:e20 doi:10.4081/cp.2013.e20

(9)Miller G, Boman J, Shrier I. (2000) Natural history of patients with adhesive small bowel obstruction. Br J Surg 87:1240-7.

(10) Garth C. Beattie, Robert T. Peters, * Susanne Guy and Richard Mendelson (2007): Computed tomography in the assessment of suspected large Bowel obstruction, ANZ J. Surg. 2007; 77: 160-165

(11) Biondo S1, Parés D, Frago R, Martí-Ragué J, Kreisler E, De Oca J, (2004). Large bowel obstruction: predictive factors for postoperative mortality. Dis Colon Rectum.;47 (11):1889-97.

(12) Ryan S., Mc Nicholas M. and Eustace S (2004). Anatomy for Diagnostic Imaging for the Abdomen and the Pelvis, Second edition; 151-245.

(13)Erik K. Paulson, and William M. Thompson, (2015) Review of Small-Bowel Obstruction: The Diagnosis and When to Worry. Radiology: Volume 275: Number 2-May radiology.rsna.org

To cite this article: Hagar A. Abdellatef, Ahmed F. Yousef, Hamada M. Khater, Adel Abdel Rahman. Role of Multi Detector CT in Diagnosis of Small and Large Bowel Obstruction. BMFJ 2021;38 (Radiology): 22-33. DOI: 10.21608/bmfj.2021.16953.1059 